\title{
Birgit Schlegel
}

\section{Umgang mit elektronischen Ressourcen am Beispiel des Dokumentenservers der Freien Universität Berlin}

DOI 10.1515/bd-2016-0038

Zusammenfassung: Nur ein geringer Teil der an wissenschaftlichen Einrichtungen publizierten Beiträge in Sammelwerken und Zeitschriften wird in der Regel auf Repositorien langzeitarchiviert.

Faktoren, wie die zum Teil geringe Kenntnis der Wissenschaftler ${ }^{1}$ auf einem hauseigenen Repositorium zu publizieren, als auch die häufig recht unzureichenden Informationen zu Urheberrecht und Vertragsgestaltung mit Verlagen (Nutzungsund Verwertungsrechte) können hierbei eine Rolle spielen.

Welche Möglichkeiten es gibt, die Dokumente einer wissenschaftlichen Einrichtung einzusammeln, welche Dienstleistungen die Universitätsbibliothek der Freien Universität Berlin in diesem Bereich anbietet, wird in dem folgenden Bericht dargestellt.

Schlüsselwörter: Akquise, SEP, Allianzlizenzen, Open Access Publikationsfonds, Zeitschriftenartikel, grüner und goldener Weg

On dealing with electronic resources, taking the example of the document server of the Freie Universität Berlin

Abstract: Only a small part of the articles published by scientists working for institutions and printed in collections and journals are regularly long-term archived in repositories.

Among the reasons could be that some scientists do not know how to publish in an in-house repository, or in many cases insufficient information about copyright and contracting with publishers (rights of use and exploitation).

1 Aufgrund der besseren Lesbarkeit wird im Text der Einfachheit halber nur die männliche Form verwendet. Die weibliche Form ist selbstverständlich immer mit eingeschlossen.

Birgit Schlegel: birgit.schlegel@fu-berlin.de 
The following article shows which opportunities there are to collect the documents of a scientific institution and which services in this field are offered by the university library of the Freie Universität Berlin.

Keywords: acquisition, SEP, alliance licences, Open Access Publishing Fund, journal article, Green and Golden Road

Neben den Hochschulschriften, die derzeit mit 10.795 Dissertationen und 634 Habilitationen den größten Anteil des Datenbestandes des DINI-zertifizierten Dokumentenservers (MyCoRe Version 2.0) bilden, umfasst der Server der FU Berlin 4.687 selbständige und unselbständige Veröffentlichungen von FU Angehörigen. Ziel ist es, alle von FU Wissenschaftlern publizierten Beiträge in Sammelwerken und Zeitschriften sowie alle selbständig erschienenen Publikationen aufzunehmen. Hierfür wurde von der Redaktion Dokumentenserver ${ }^{2}$ der Universitätsbibliothek ein Geschäftsgang etabliert, mit dessen Hilfe Publikationen von FU Angehörigen kontinuierlich als Erst- oder Zeitkopie im Dokumentenserver langzeitarchiviert werden.

Zur effizienten Abwicklung all der in diesem Zusammenhang nötigen Tätigkeiten bedient sich die Redaktion Dokumentenserver des Open Ticket Request Systems (OTRS) ${ }^{3}$, eines webbasierten Helpdesk-Systems, mit dem alle Kundenanfragen per E-Mail oder Telefon strukturiert erfasst, klassifiziert gespeichert und anschließend weiterverarbeitet werden. Alle Mitarbeiter haben jederzeit Zugriff auf den Bearbeitungsstand der einzelnen Vorgänge innerhalb der jedem Nutzer zugewiesenen Queue. Dies gewährleistet eine zeitnahe und strukturierte Abarbeitung der eingehenden Anfragen unter Verwendung spezieller Antwortvorlagen. Über sogenannte Erinnerungstickets steuert die Redaktion Dokumentenserver die regelmäßige Überprüfung auf Neuerscheinungen von FU Publikationen.

2 http://edocs.fu-berlin.de/docs/content/main/contact.xml [Zugriff: 22.01.16].

3 http://www.otrs.com [Zugriff: 22.01.16]. 


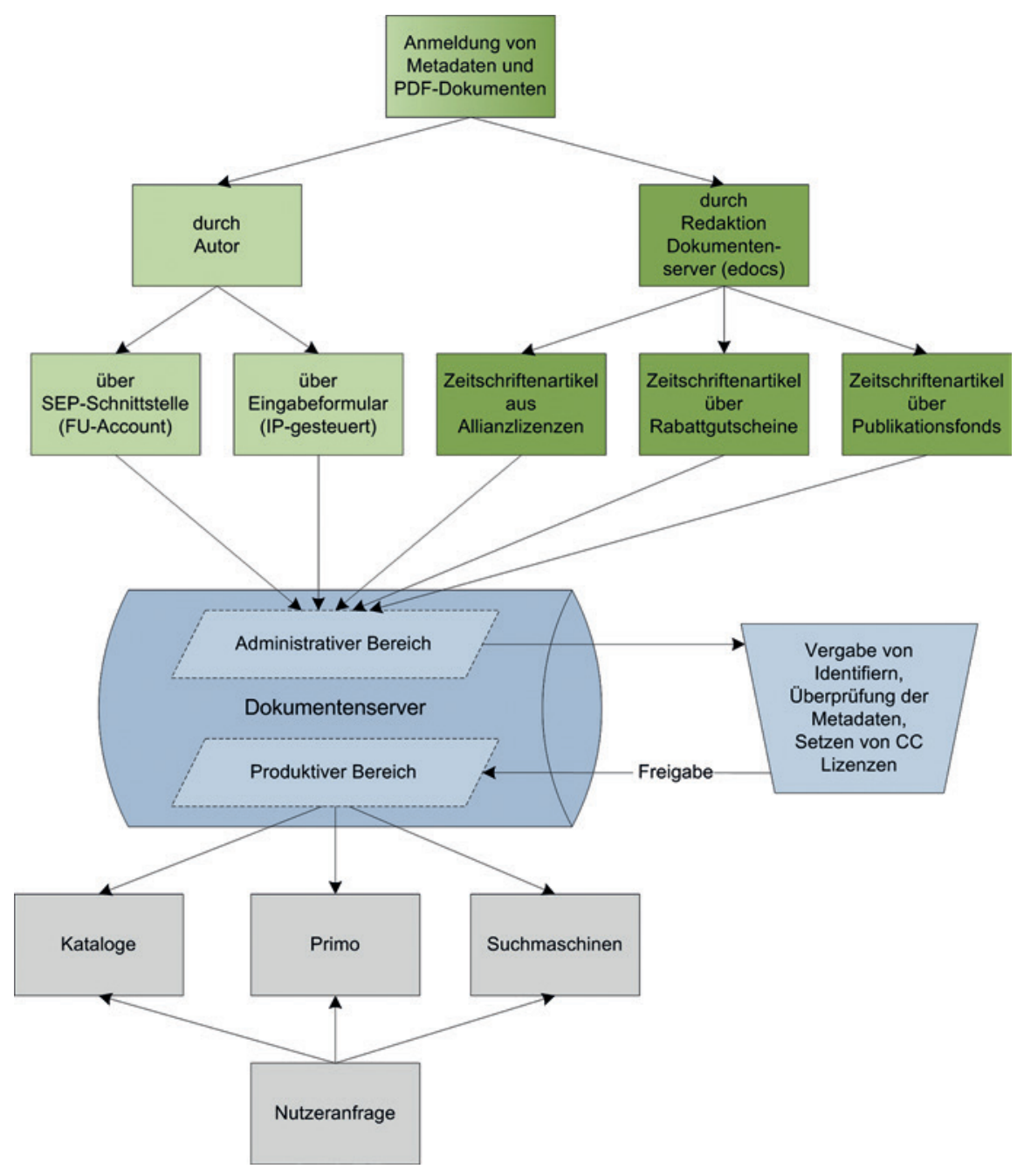

Abb. 1: vereinfachte Darstellung des Geschäftsgangs.

Die Eingabe der Metadaten in den Server erfolgt seitens der Autoren entweder über die SEP-Schnittstelle oder direkt über das Eingabeformular des Dokumentenservers. 
Seit 2010 existiert das System zur Selbsterfassung der Publikationen für Angehörige der Freien Universität Berlin (SEP) ${ }^{4}$. Voraussetzung für die Publikationserfassung in SEP ist ein FU-Account. Über die bibliographisch korrekten Publikationsnachweise kann die leistungsbezogene Mittelverteilung der Universität erfolgen. Gleichzeitig wird durch die Eingabe der Daten die Universitätsbibliographie gespeist. Anfang 2014 wurden neue Open Access Felder sowie eine Schnittstelle zum Dokumentenserver in SEP integriert. Die Felder erlauben eine spezifische Auswertung der Open- Access Publikationen incl. der eventuell angefallenen Publikationsgebühren. Über die implementierte SEP-Schnittstelle erhält der Dokumentenserver Metadaten von Publikationen.

Nach erfolgter Selbsteingabe der Publikationsdaten in SEP hat der Autor zwei Möglichkeiten. Er wendet sich per Mausklick (E-Mail) an die Redaktion Dokumentenserver, die weitere Hilfestellung leistet, oder er betätigt den Hochladebutton. Die zuvor eingegebenen Metadaten werden nun über die SEP-Schnittstelle im administrativen Bereich des Dokumentenservers angelegt.

Wählt der Wissenschaftler die direkte Eingabemaske ${ }^{5}$ in den Dokumentenserver, erfolgt eine IP- und Zeitstempel gesteuerte Eingabe der Metadaten mit einigen wenigen Pflichtfeldern. Per Mausklick wird der im System hinterlegte Veröffentlichungsvertrag ${ }^{6}$ akzeptiert. Von der Möglichkeit der selbständigen Anmeldung der Dokumente durch die Autoren wird seit 2012 zunehmend Gebrauch gemacht. $\mathrm{Zu}$ dieser Steigerung des Bekanntheitsgrads des Dokumentenservers hat zweifelsohne die Einführung des Open-Access Publikationsfonds beigetragen.

Alle eingegebenen Metadaten und Dateien gelangen zunächst in den Administrativbereich des Servers und werden hier in unterschiedliche Queues zur Weiterbearbeitung abgelegt.

Die folgenden Arbeitsschritte der Redaktion Dokumentenserver schließen nun routinemäßig an.

- Bei Vorliegen einer Erstkopie Nachfrage bei Autoren, ob eine ISBN oder ein DOI, ggf. eine ISSN vergeben werden soll. Die Universitätsbibliothek fungiert seit Anfang 2015 als Datenzentrum für die DOI Vergabe für alle Einrichtungen der Freien Universität Berlin.

- Überprüfung der Metadaten und der PDF-Datei auf formale Kriterien und Vollständigkeit, ggf. Rücksprache mit Autor/Fachbereich,

- bei erfolgter Anmeldung über die SEP Schnittstelle ggf. Anforderung der PDF-Dateien, sofern der Autor lediglich die Metadaten übermittelt hat,

4 https://sep.ub.fu-berlin.de/SEP/ [Zugriff: 22.01.16].

5 http://edocs.fu-berlin.de/docs/content/main/autoren/Menu.xml [Zugriff: 22.01.16].

$6 \mathrm{http}$ ://edocs.fu-berlin.de/docs/content/main/autoren/vertraege.xml [Zugriff: 22.01.16]. 
- Nutzung der Check Copyright Funktion im Metadatenformular zur Weiterleitung zur Sherpa Romeo Liste. Die allgemeinen Konditionen gilt es hier zu beachten, ggf. Klärung von Verlagsrechten, Beratung der Autoren in urheberrechtlichen Fragen.

- Prüfung und Korrektur der Metadaten, formale Durchsicht der PDF-Datei,

- Anpassung der Nutzungsbedingungen, Setzen von CC- Lizenzen, sofern vorhanden oder eines Links auf das Verlagscopyright in eigens dafür zur Verfügung stehenden Metadatenfeldern,

- Freischaltung der Dokumente für den Produktivbereich des Dokumentenservers (Preprint oder Verlagsfassung),

- E-Mail-Benachrichtigung an die Autoren unter Angabe der URL des freigeschalteten Dokuments,

- Nachmeldung der Publikationen der letzten zwei Jahre an SEP, sofern noch kein Eintrag erfolgt ist,

- Nachweis aller Publikationen in Discovery-Systemen, Suchmaschinen und Bibliothekskatalogen über die Z3950 Schnittstelle.

Um den Arbeitsaufwand für die Wissenschaftler möglichst gering zu halten, bietet ihnen die Redaktion insbesondere bei periodisch erscheinenden Veröffentlichungen an, die Eingabe von Metadaten und Dateien für sie zu übernehmen. Zumeist handelt es sich hierbei um Schriftenreihen, für die zwischen der Universitätsbibliothek und dem Autorenkollektiv oder Herausgeber ein Herausgebervertrag geschlossen wurde. Im Fall einer Neuerscheinung erinnert ein zuvor gesetztes OTRS Ticket an das Einpflegen neuer Dokumente. Seit Einführung der Herausgeberverträge in 2012 konnte die Zahl der eingeworbenen Schriftenreihen verdoppelt werden. Das Angebot der Eingabe der Metadaten durch die Redaktion gilt gleichermaßen für Erstattungsanträge im Rahmen des Publikationsfonds, auf die im weiteren Verlauf noch detailliert eingegangen wird.

Gemäß der Open Access Komponente in den DFG geförderten überregionalen Lizenzen ${ }^{7}$ sind „Autoren aus autorisierten Einrichtungen ohne Mehrkosten berechtigt, ihre in lizenzierten Zeitschriften erschienenen Artikel in der Regel in der durch den Verlag publizierten Form (z. B. PDF) zeitnah in institutionelle oder disziplinspezifische Repositorien ihrer Wahl einzupflegen und im Open Access zugänglich zu machen. Das gleiche Recht besitzen die autorisierten Einrichtungen, denen die jeweiligen Autoren angehören“.

Um alle in den vergangenen Jahren im Rahmen der Allianzlizenzen publizierten Artikel von FU-Wissenschaftlern in den Dokumentenserver in Zweitkopie

7 http://www.dfg.de/formulare/12_181/12_181_de.pdf [Zugriff: 22.01.16]. 
(grüner Weg) aufzunehmen, erstellt die Redaktion in regelmäßigen Abständen Alerts in Web of Science Core Collection und Scopus (Suchanfrage: Index: Organization-Enhanced, Suchbegriff: „Free University of Berlin“, Einschränkung auf Document Type =Article, ggf. Einschränkung auf ,Time Span`).

Die recherchierten Zeitschriftenartikel werden unter Abgleich der Lizenzlaufzeit und der Frage, welche Version in den Dokumentenserver übernommen werden darf, geprüft. Ob es ein Embargo von sechs oder zwölf Monaten gibt, ob lediglich ein Post- oder Preprint verwendet werden darf, hängt von den in den Allianzverträgen ausgehandelten Bedingungen ab.

Zusätzlich klärt die Redaktion, ob es sich bei den jeweiligen Artikeln um erstattungsfähige Anträge im Rahmen des Publikationsfonds ${ }^{8}$ handelt. Sollte dies der Fall sein, werden die Autoren über diese Möglichkeit per OTRS-Emailticket informiert.

Der Metadatensatz erhält in dafür vorgesehenen Feldern eine entsprechende Kennzeichnung (Publikationsfonds ja/nein, Antrag gestellt). Nach abschließender Prüfung passt die Redaktion die Hinweise in den Metadatenfeldern an und lädt den vervollständigten Metadatensatz samt PDF-Datei in den Server.

Nicht für alle Verlage oder Anbieter sind die Recherchen nach FU-Publikationen in Web of Science oder Scopus erfolgreich. Einige Verlage wie Karger Journals, Wiley AGU oder China Academic Journals werden von der Redaktion direkt angeschrieben. In der Regel senden diese Verlage dann Publikationslisten der betreffenden Jahrgänge zu, oder sie stellen der Redaktion eine adäquate Suchanfrage über ihre Verlagswebseite zur Verfügung. Bei wieder anderen Verlagen wie Cambridge Journals recherchiert die Redaktion selbständig auf den Verlagsseiten. Verlage wie De Gruyter unterstützen die Redaktion Dokumentenserver bei der Nutzung der Open Access Komponente, indem sie eine Sword Schnittstelle zur Verfügung stellen. Damit ist eine automatisierte Einspielung von Zeitschriftenartikeln der De Gruyter Online-Journals / Linguistics, Literature and Humanities (LLH) 2011-2013 und 2014 in den Dokumentenserver gewährleistet.

Einige Verlage bieten teilnehmenden Einrichtungen wie der Freien Universität Berlin Rabatte oder Gutscheine bei Publikationsgebühren an. Im Rahmen dieser sogenannten Allianzlizenzen können Publikationen über den goldenen Weg direkt Open Access gestellt werden. Die Open-Access-Token der Royal Society of Chemistry (RSC) ${ }^{9}$ sind ein Beispiel dafür. Die Redaktion Dokumentenserver

8 http://www.fu-berlin.de/sites/open_access/dienstleistungen/artikelgebuehren/publikationsfonds/[Zugriff: 22.01.16].

$9 \mathrm{http://www.rsc.org/[Zugriff:} \mathrm{22.01.16].}$ 
stellt FU-Autoren des Fachbereichs Biologie, Chemie, Pharmazie die Gutscheine in regelmäßigen Abständen zur Verfügung.

Über einen in der E-Mail an den Autor angegebenen Voucher-Code gelangen die Autoren zum Anmeldeformular der RSC. Voucher Nummer und Institution werden bei Benutzung eines Voucher Codes automatisch angegeben. Die Autoren vervollständigen das Webformular und senden es ab. Der License to Publish (CC-BY oder CC-BY-NC) stimmen sie dabei online zu. Sobald die Bestätigung über die Einlösung des Gutscheins bei der Redaktion Dokumentenserver eintrifft, erscheint der Artikel kostenlos und ohne Zeitverzögerung auf der Seite des Verlages Open Access und kann auf diese Weise in den Dokumentenserver übernommen werden. Die Angabe der Token-Nummer in den Metadatenfeldern gewährleistet eine statistische Auswertung.

Der Open-Access-Publikationsfonds (goldener Weg) wurde 2012 aus Mitteln der Deutschen Forschungsgemeinschaft (DFG) und der Freien Universität Berlin eingerichtet. Aus diesem Fonds, den die Universitätsbibliothek verwaltet, werden Artikel finanziert, die von FU-Angehörigen in Open-Access Zeitschriften veröffentlicht werden und die die von der DFG formulierten Bedingungen für die Kostenübernahme erfüllen. Im Februar 2013 hat das Präsidium der Freien Universität Berlin entschieden, den Fonds dauerhaft, d. h. auch nach Auslaufen des DFGProgramms 2020 für Universitätsangehörige vorzuhalten. Als positiver Nebeneffekt wird jeder erstattungsfähige Artikel nach seinem Erscheinen zusätzlich über den Dokumentenserver weltweit zur Verfügung gestellt.

Die Beantragungsmodalitäten sind unkompliziert gestaltet. Der Antragsteller (corresponding author) füllt das auf der Webseite zur Verfügung stehende Formular ${ }^{10}$ aus. Am Ende des Formulars wird er interaktiv weitergeleitet zum Dokumentenserver, wo er die Möglichkeit hat, Metadaten und Dokument anzumelden. In den Fällen, in denen der Artikel noch nicht erschienen ist, legt die Redaktion für den Autor zunächst einen Metadatensatz an, den sie später durch das PDF Dokument vervollständigt. Sobald alle erforderlichen Unterlagen, insbesondere die einzureichenden Kontoauszüge sowie das korrekt ausgefüllte Antragsformular vorliegen, leitet die Redaktion Dokumentenserver den Antrag zur Erstattung an die Verwaltung der Universitätsbibliothek weiter. Metadaten und PDF-Datei des Dokuments verbleiben bis zur Erstattung der Anträge im administrativen Bereich des Dokumentenservers. Bei der Überprüfung der Metadaten werden diese Veröffentlichungen durch das Setzen der CC-Lizenz und entsprechender Vermerke zum Publikationsfonds in den dafür zur Verfügung stehenden Metada-

$10 \mathrm{http} / / / w w w . f u-b e r l i n . d e / s i t e s / o p e n \_a c c e s s / d i e n s t l e i s t u n g e n / a r t i k e l g e b u e h r e n / p u b l i k a t i-$ onsfonds/voraussetzungen/index.html [Zugriff: 22.01.16]. 
tenfeldern gekennzeichnet. Diese Felder dienen u. a. der Auswertung der Anzahl der erstatteten Anträge pro Jahr. Nach erfolgter Erstattung erhält der Autor eine Emailbenachrichtigung über den Abschluss der Arbeiten unter Angabe der URL des freigeschalteten Artikels.

Die Anzahl der erstatteten Anträge konnte seit Einführung des Fonds im Jahr 2012 in 2013 um 112\% und in 2014 gegenüber dem Vorjahr um 38\% gesteigert werden, für 2015 ist eine weitere Steigerung von 42\% gegenüber 2014 zu erwarten.

Seit 2014 nimmt die Universitätsbibliothek am Projekt Datasets on fee-based Open-Access Publishing der Uni Bielefeld ${ }^{11}$ teil.

Hinsichtlich der geschilderten Aktivitäten der Akquise von Dokumenten der FU-Angehörigen verdeutlicht die folgende Grafik den Zuwachs an Dokumenten in den Jahren 2012-2015. Nennenswert ist die Steigerung von $41 \%$ in 203 und von 92\% in 2014 im Vergleich zum Vorjahr.

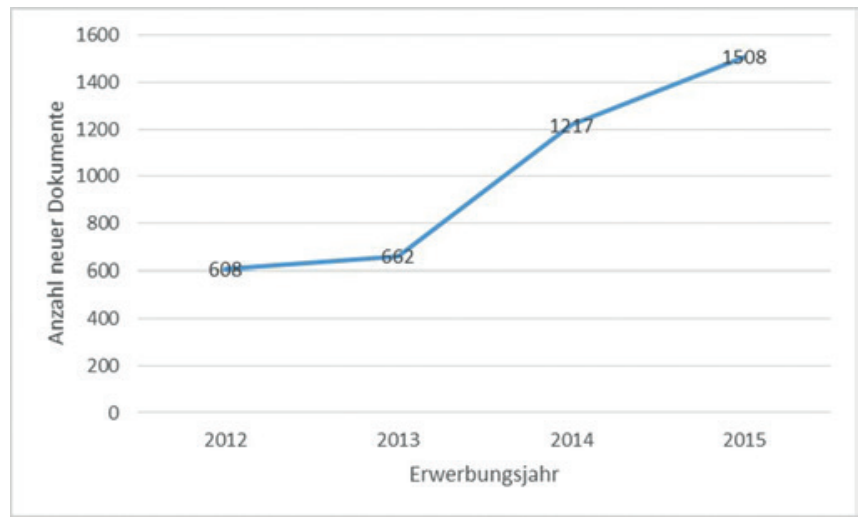

Abb. 2: Steigerung der Akquise ab 2012.

Die Universitätsbibliothek/Redaktion Dokumentenserver wird ihre Angebote weiter professionalisieren. Dazu gehören u.a. die Digitalisierung gemeinfreier Ressourcen, der Abschluss institutioneller Mitgliedschaften mit Verlagen sowie das Angebot der Direktbezahlung bei Artikelgebühren. In der Umsetzungsphase begriffen ist die Übernahme der über die Software Open Journals Systems (OJS) des Centers für Digitale Systeme (CeDiS) ${ }^{12}$ gehosteten Zeitschriften in den Doku-

11 https://github.com/OpenAPC [Zugriff: 22.01.16].

12 http://www.cedis.fu-berlin.de/ [Zugriff: 22.01.16]. 
mentenserver. Hinsichtlich der Abstimmung eines gemeinsamen Serviceangebotes für FU Wissenschaftler sind erste Schritte zur Anwendung gemeinsamer Herausgeberverträge getan.

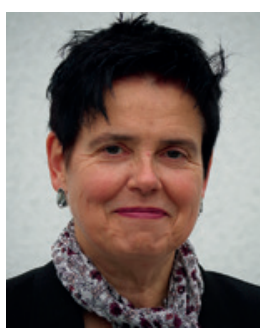

\section{Birgit Schlegel (Dipl. Bibl.)}

Freie Universität Berlin

Universitätsbibliothek

Hochschulschriftenstelle und Dokumentenserver

Garystr. 39

14195 Berlin

E-Mail: birgit.schlegel@fu-berlin.de 